\title{
Defects in nano-imprint lithography line patterns: computational modelling and measurement accuracy
}

Constantoudis, Vassilios, Whitworth, Guy, Kehagias, Nikolaos, Papavieros, George, Sotomoyor Torres, Clivia, et al.

Vassilios Constantoudis, Guy Whitworth, Nikolaos Kehagias, George Papavieros, Clivia M. Sotomoyor Torres, Evangelos Gogolides, "Defects in nano-imprint lithography line patterns: computational modelling and measurement accuracy," Proc. SPIE 10958, Novel Patterning Technologies for Semiconductors, MEMS/NEMS, and MOEMS 2019, 109581K (26 March 2019); doi: 10.1117/12.2523931

SPIE. Event: SPIE Advanced Lithography, 2019, San Jose, California, United States 


\title{
Defects in Nano-imprint Lithography Line Patterns: Computational Modelling and Measurement Accuracy
}

\author{
Vassilios Constantoudis ${ }^{1,2, *}$, Guy Whitworth ${ }^{4}$, Nikolaos Kehagias ${ }^{4}$, George Papavieros ${ }^{1,2,3}$, Clivia \\ M. Sotomoyor Torres ${ }^{4,5}$, and Evangelos Gogolides ${ }^{1,2}$ \\ ${ }^{1}$ Institute of Nanoscience and Nanotechnology, N.C.S.R. Demokritos, Agia Paraskevi, Greece \\ ${ }^{2}$ Nanometrisis p.c., Agia Paraskevi, Greece \\ ${ }^{3}$ Physics Department, Aristotle University of Thessaloniki, Thessaloniki 4124, Greece \\ ${ }^{4}$ Catalan Institute of Nanoscience and Nanotechnology (ICN2), CSIC and BIST, \\ Campus UAB, Bellaterra, 08193 Barcelona, Spain \\ ${ }^{5}$ ICREA, Institució Catalana de Recerca i Estudis Avançats, 08010 Barcelona, Spain
}

*v.constantoudis@inn.demokritos.gr

\begin{abstract}
NIL patterns frequently suffer from the presence of defects such as missing lines or dots which degrade their properties and functionality. Due to their low density and nanosize, the measurement of their fraction is challenging nanometrology trade-off between resolution and measurement range. In this paper, we focus on the use of range-limited SEM images and explore the benefits of a computational modeling approach to simulate the measurement process and estimate the statistics and accuracy of the measurement of missing lines in patterns. The main questions we address have to do with the choice of the parameters available in the measurement process such as the number of acquired images, their magnification defining the lines included in images and the position (overlapped or not) at line pattern. The missing lines can have both uncorrelated and correlated positions in pattern. In the case of positive correlations, the defects are aggregated whereas in the opposite case of negative correlations they are arranged in periodic-like positions. We found that for uncorrelated defects, the critical parameter is the total number of lines included in the measurement process while the image position do not have any impact on the measurement accuracy. On the contrary, when correlations in defect positions are considered, the number of images and the number of lines per image differentiate their effects on the accuracy of the result while the arrangement of images along pattern also plays a crucial role in the measurement process.
\end{abstract}

Keywords: Defect metrology, NIL patterns, statistical modelling, measurement protocol, SEM images, correlations

\section{INTRODUCTION}

Line patterns produced by Nanoimprint Lithography (NIL) may suffer from a wide spectrum of defects ${ }^{1}$. One of the most commonly encountered type of defects is the missing lines at some positions of the line pattern which can be also called non-fill or gap-associated defects ${ }^{1,2}$. Not surprisingly, the quality control of a patterning process should contain the measurement of the fraction of defects in the pattern (i.e. their density) to justify their performance. The measurement of the fraction of missing lines can be done either by indirect optical techniques (i.e. scatteromettry) $)^{3,4}$ or with more direct

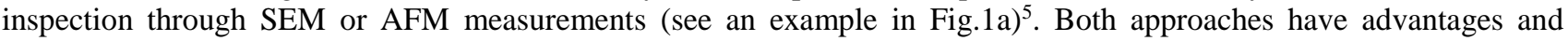
drawbacks. The measurement range of the first approach covers a large area of pattern with thousands of lines to guarantee the statistical reliability of the measurement and are working inline but the results are indirect based on hypotheses and models. On the other hand, the scanning microscopy (SEM /AFM) techniques are imaging a limited number of lines from a local segment of the whole pattern area making the measurement of defect fraction uncertain though their application is more straightforward with more direct interpretation. The variation of SEM measurements of defect fraction is explicitly shown in Fig.2, where a line pattern with missing lines is shown along with the positions of some (4) SEM images enclosing

Novel Patterning Technologies for Semiconductors, MEMS/NEMS, and MOEMS 2019,

edited by Martha I. Sanchez, Eric M. Panning, Proc. of SPIE Vol. 10958, 109581K

(c) 2019 SPIE · CCC code: $0277-786 X / 19 / \$ 18 \cdot$ doi: $10.1117 / 12.2523931$

Proc. of SPIE Vol. 10958 109581K-1 
segments of the whole pattern. As one can easily see, the measurements of four images derive very disperse results ranging from 1 defect in SEM image 1 (fraction=0.06) to 7 missing lines in SEM image 3 (fraction=0.44).

In order to compare the results of the aforementioned two approaches, we should elaborate the local SEM measurements to make inferences for the limit of the large-scale measurements of the optical techniques. The questions which should be addressed are the uncertainty of the SEM measurements when compared with optical results and the measurement protocol we should apply in order to get results from SEM inspection with uncertainties below some predetermined value. Furthermore, we can investigate the correlations among the positions of the missing lines and how these can be estimated through the analysis of SEM images.

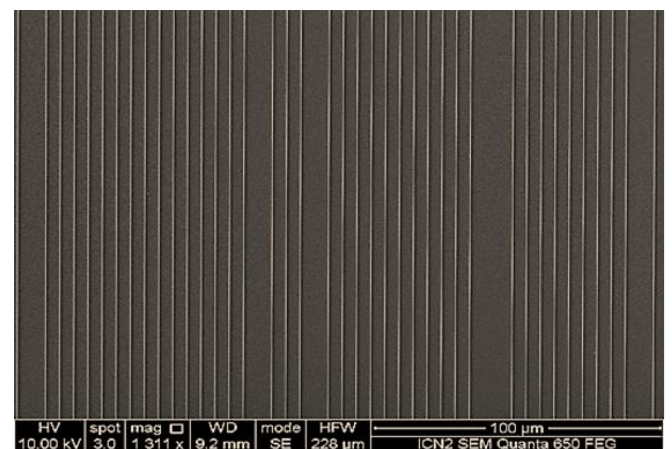

(a)

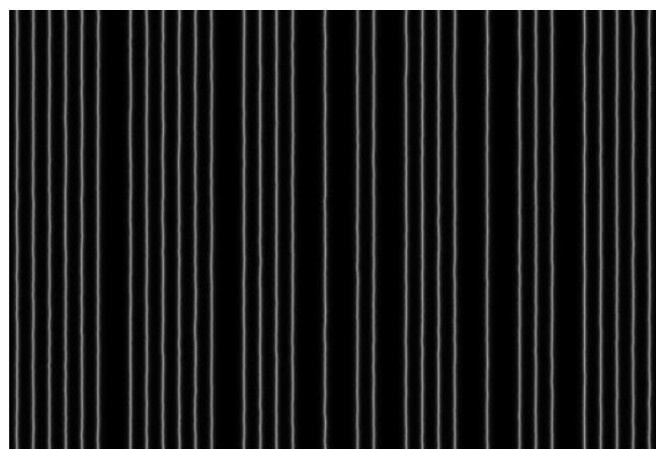

(b)

Fig. 1. a) Experimental SEM image of a real line pattern with missing lines as defects, b) Synthesized SEM image of a model line pattern with missing lines

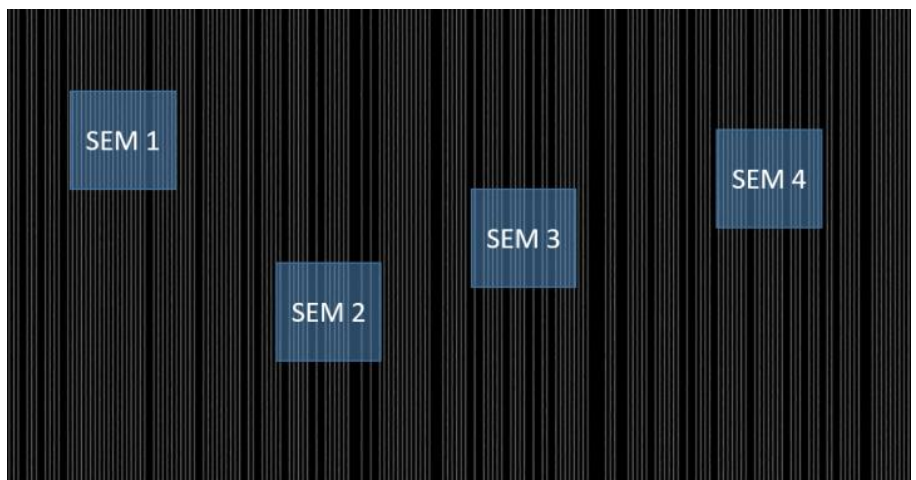

Fig. 2. Schematic of a large area of a line pattern with defects of the type of missing lines and some indicative areas of SEM images acquired to measure the fraction of defects. One can notice the wide variation of the measured defects in each SEM image (from 1 in SEM 1 to 6 in SEM 2) which clearly demonstrates the need for a deeper investigation of the statistics of defect measurements aided by modelling.

The aim of this paper is to investigate the above questions through the computational modeling of the large-scale pattern with the missing lines and the local scale SEM image areas. First, we assume no correlations among missing line positions and calculate the uncertainty of the measured defect fraction versus the number of SEM images and their magnification (measurement range). Both large and small fractions of defects are considered. The final output of the simulations is to build a protocol for SEM measurements with controlled uncertainty so that to make feasible the comparison with the largescale optical techniques. Secondly, we impose correlations between the positions of missing lines in the pattern and we quantify their footprint in SEM measurements, uncertainty and protocol. The correlations can be positive leading to the formation of defect aggregations randomly distributed in the pattern or can be periodic-like positioning the defects in aggregates with periodic arrangements in the pattern. We consider the case of defect correlations since in real patterns with sub $<20 \mathrm{~nm}$ nanostructures, the patterning process and its failure scannot be limited in the area of a sole nanostructure. 
The paper is structured as follows. In the next section 2, we present the modeling approach and the steps for its implementation. The results are reported in Section 3 for both uncorrelated (3.1) and correlated (3.2) defect positions. The main conclusions of the results are summarized in the final section 4.

\section{MODELING METHODOLOGY}

The aim of our modelling method is to provide a tool to estimate the uncertainty of the SEM measurements of the defects of a NIL pattern. The methodology is general and can be applied to any kind of pattern (dot, hole or line) but here we focus on line patterns and in the case where the defects are missing lines. The steps of our computational modelling of the statistics of SEM measurements are shown below:

1. First, we generate a line pattern covering a large macroscopic area and containing a very large number of lines $\mathrm{N}$ simulating the real NIL nanostructured patterns where due to the small pitch one can enumerate millions or even billions of nanostructures in the patterned area. For example, in an area $1 \mathrm{~m}^{2}$ patterned with lines having pitch $100 \mathrm{~nm}$, one will have $10^{7}$ lines across it. This is why in our modeling runs we choose $\mathrm{N}=10^{7}$. The number of missing lines $\mathrm{N}_{\text {def }}$ is determined by the defect fraction $\mathrm{p}_{\text {def }}$ from the relationship $\mathrm{N}_{\text {def }}=p_{\text {def }} \mathrm{N}$.

2. In order to get a convenient math representation of the pattern targeted to defect metrology, we transform the pattern with the defects in a digital symbolic series where $1 \mathrm{~s}$ stand for lines and 0s for the missing lines. This transformation enables the mathematical and computational treatment of defect statistics and empowers our analysis with the rich spectrum of methods for binary series analysis ${ }^{6}$.

3. Then the acquisition of SEM images is modelled with the selection of local segments of the whole digital series representing the total pattern. The SEM metrologist controls three parameters of this process besides the conventional SEM settings: the number of images M, the magnification of the image which defines the number of lines $\mathrm{K}$ including in each image and the positions of the segments imaged by SEM measurements. The latter can be randomly selected or can be programmed so that SEM images do not include the same lines. In the first case, we are talking about images with overlapping lines whereas the second case is named nonooverlapping.

4. After the selection of the input parameters ( $N$, $\mathrm{p}_{\mathrm{def}}, \mathrm{M}, \mathrm{K}$, (non)overlapping), we perform the statistical analysis as follows: for each specific couple $\mathrm{M}, \mathrm{K}$ we calculate the standard deviation of the mean values of defect fractions as they are estimated by a large number of repetitions of the measurement process for different choices of the $\mathrm{M}$ SEM image positions. The obtained standard deviation is normalized to the total mean of defect fractions which is very close to the true input value $p_{\text {def. }}$ The goal here is to give an estimation of the error of the measurement when M SEM images with K lines in each are employed.

5. The output of statistical analysis is presented in the form of contour plots of the normalized standard deviation versus number of lines $\mathrm{K}$ and number of images $\mathrm{M}$. These results can be used to build a measurement protocol for SEM-based measurements of defects enabling the reliable comparison with large-scale optical measurements.

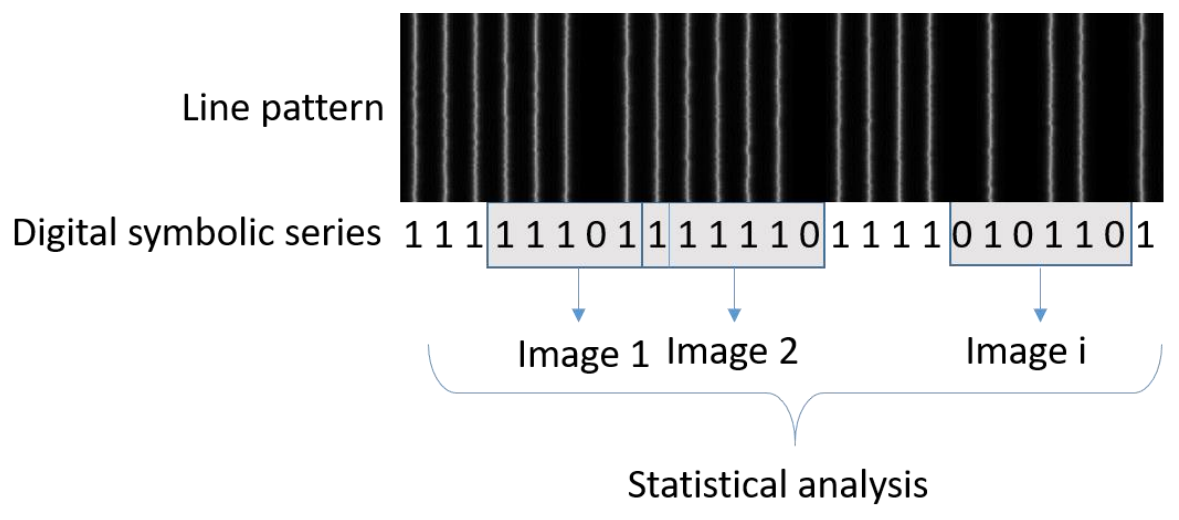

Fig. 3. The key idea of our computational modelling method: first the line pattern is transformed to a digital symbolic series where 1 stands for a line and 0 for the missing lines (gaps) and then segments of this series is statistically analysed to simulate the SEM image acquisition and results. 


\section{RESULTS}

The steps of our modelling methodology describing in the previous section have been applied for three different values of defect fractions $(0.1,0.05$ and 0.01$)$ of the whole line pattern. For each value, three cases were considered where the positions of the defects (missing lines) have been uncorrelated, positively correlated grouping in aggregates randomly distributed along the pattern and negatively correlated in groups repelling each other in almost equal distance defined by the period. Concerning the measurement process, the impact of the position of SEM images (overlapping or nonoverlapping) on measurement accuracy has been examined. For the sake of clarity, Table 1 summarizes the cases we considered along with the notation used in the presentation of the results in the following sub-sections.

\begin{tabular}{|c|c|c|c|}
\hline & A. Uncorrelated & B. Aggregated & C. Periodic-like \\
\hline 1. Overlapping images & 11101111011111010111 & 11100011111001111100 & 11101110111011101101 \\
\hline 2. Non-overlapping images & 11101111011111010111 & 11100011111001111100 & 11101110111011101101 \\
\hline
\end{tabular}

Table 1. Presentation of the cases investigated in the paper. The defect positions can be uncorrelated or positively correlated (aggregated) or negatively correlated (periodic-like) while the acquisition of the SEM images can be with (overlapping) and without (non-overlapping) including the same lines

\subsection{Uncorrelated defects}

The results of the computational modelling for the uncorrelated defects (case A) are displayed in Fig. 4. The first row contains the contour graphs of the normalized standard deviation of the calculated defect fractions vs. the number of lines $\mathrm{K}$ and the number of images $\mathrm{M}$ when the images are taken randomly and for three different values of real defect fractions: a) $\left.\mathrm{p}_{\mathrm{def}}=0.1, \mathrm{~b}\right) \mathrm{p}_{\mathrm{def}}=0.05$ and c) $\mathrm{p}_{\mathrm{def}}=0.01$. The second row presents similar results for the case of nonoverlapping images.

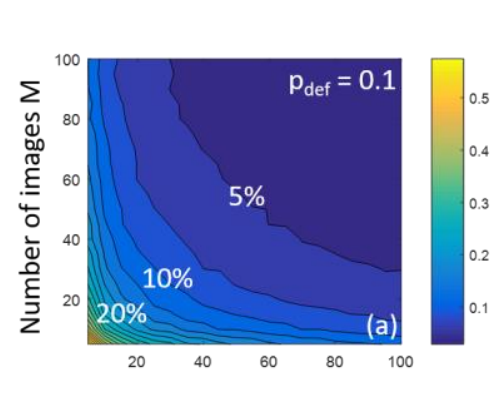

A1 (Uncorrelated - overlapping)
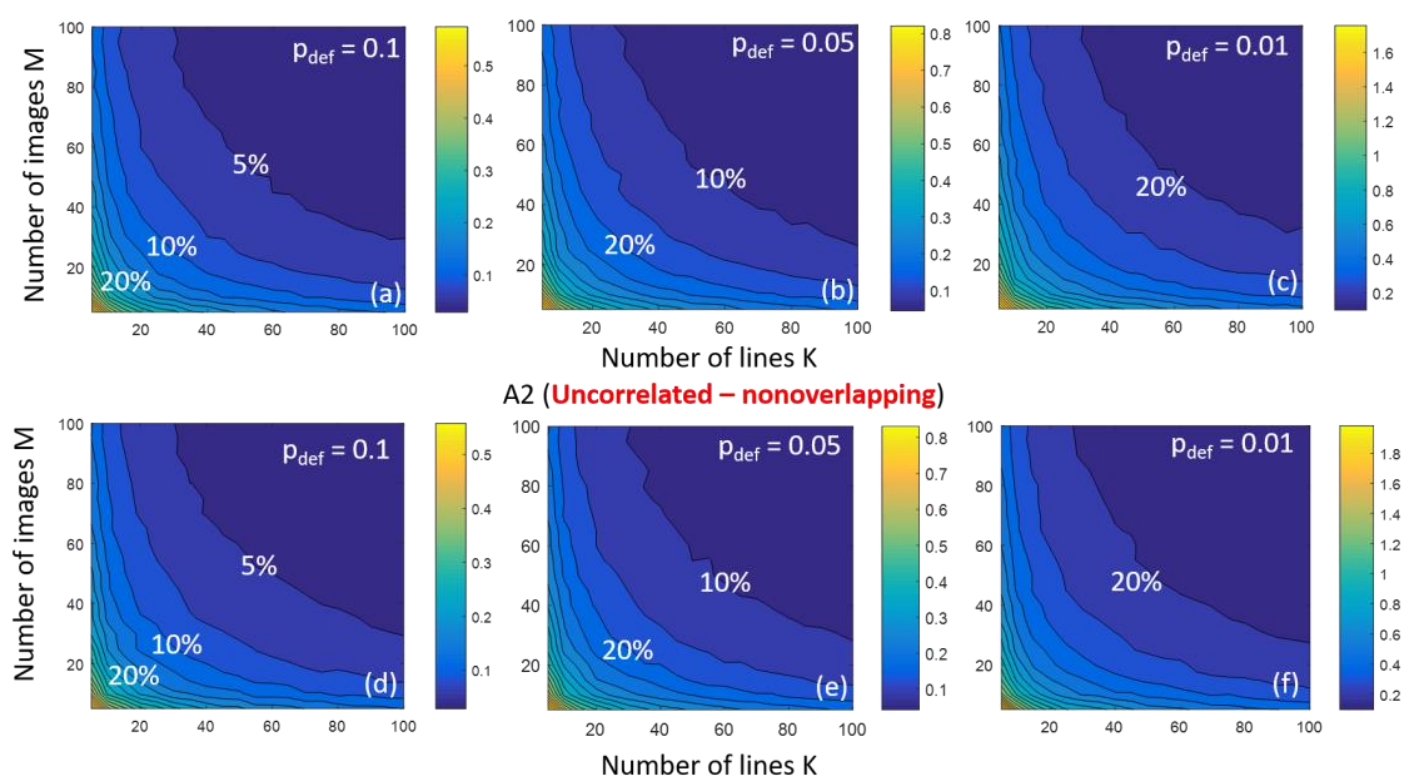

Fig. 4. Contour graphs of the normalized standard deviation of the fraction of missing lines versus the number M of acquired SEM images and the number $\mathrm{K}$ of lines contained in these when the positions of missing lines in the pattern are uncorrelated. The first row (a-c) show the results of our statistical modelling when the images may include the same lines (overlapping) (case A1) while the second (d-f) the results for the case A2 where the images are imposed to include different lines. The columns correspond to different fraction of defects in the whole pattern: $\mathrm{p}_{\text {def }}=0.1(\mathrm{a}, \mathrm{d}), \mathrm{p}_{\mathrm{def}}=0.05(\mathrm{~b}, \mathrm{e})$ and $\mathrm{p}_{\mathrm{def}}=0.01(\mathrm{c}, \mathrm{f})$. 
One can easily notice that in all plots, the contour lines have the form of $1 / \mathrm{x}$ which means that the standard deviation does not depend separately from $\mathrm{M}$ and $\mathrm{K}$ but from their product $\mathrm{M}^{*} \mathrm{~K}$ which seems to be the critical parameter. Secondly, there is no difference between the plots of the first and second row at the same $p_{\text {def. }}$ This is in accordance with the sampling theory according to which for sufficiently large populations (pattern size) and randomness the sampling with replacement or no replacement (overlapping and nonoverlapping in our terminology) does not have significant effect on the measurands. Finally, as expected the uncertainty goes up drastically at low $\mathrm{p}_{\text {def }}$ where uncertainties lower than $20 \%$ requires the acquisition of more than 10 times images.

\subsection{Positively Correlated (aggregated) defects}

By correlations we mean that the position of each defect can affect the positions of their neighbours. In general, we can have two types of correlations: positive and negative. In the first case of positive correlations, the presence of a defect at a specific pattern location increases the probability to have other defects in its neighbourhood. This results in an increased trend to get in aggregations of defects with probability larger than that of Poisson randomness. The mean size of aggregates is dictated by the correlation length of defect positions, i.e. the range along line pattern of the interaction between defects.

Fig. 5 and Fig. 6 show the results of our modelling calculations for the standard deviation of defect fraction for two correlation lengths 5 and 20 pitches respectively simulating the cases of short and long range positive correlations between defects. The main question of our investigation concerns the impact of these correlations in the accuracy of defect metrology.

As shown in the contour graphs of Figs 5 and 6, there are two differentiations with respect to the behavior reported in the uncorrelated case A:

a) The image acquisition process seems to have a slight effect on the measurement accuracy with the nonoverlapping process to output larger standard deviations especially at small $\mathrm{M}, \mathrm{K}$. This means that when for some reason we expect aggregated defects, it is preferable to select image positions randomly with no concern about the consideration of the same lines in the measurement process.

b) The symmetry between $\mathrm{M}$ and $\mathrm{K}$ is broken down: to get more images has more dramatic effects than the increase of lines per image in all correlation lengths and image acquisition processes.

Both differentiations can be explained based on the increased heterogeneity of defect dispersion in pattern associated with correlations. The reliable statistical representation of this heterogeneity favours the acquisition of randomly obtained several images with small number of lines instead of getting more programmed images with several lines in each.

\subsection{Negatively correlated (periodic-like) defects}

In the case of negative correlations, the defects (missing lines) are positioned in the pattern following an almost periodic arrangement. They can be isolated or gathered in groups. In both cases, they are almost equally spaced in the pattern with their mean distance defined by the period. In our computational modelling approach, we consider group of defects with size proportional to their period in order to get patterns with defect distributions spatially characterized by a single parameter.

The results for a period equal to 100 pitches are shown in Fig. 7 again in the form of contour graphs of the standard deviation of defect fractions versus the number of images and lines. Here, the impact of the way we acquire the images is catalytic. When we arrange the image positioning to avoid overlapping then a resonancelike behavior occurs at submultiples of the period where the standard deviation increases steeply. In between, we can have low uncertainties even at small number of images and lines.

Furthermore, a noteworthy change in the direction of contour lines is observed for the overlapping case: contrary to what is happening with aggregated defects, here the contour lines are becoming more vertical leading to the conclusion that it is more preferable in measurements to get fewer images with more lines in each of these. 


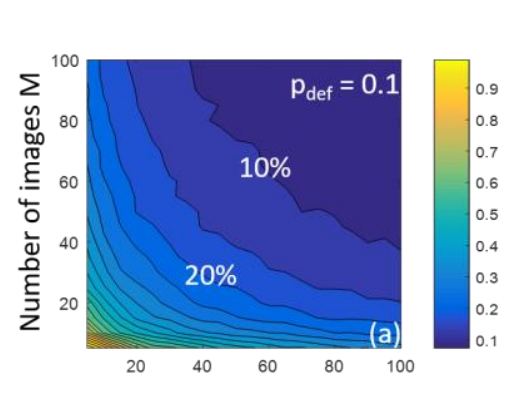

B1 (aggregated- overlapping)

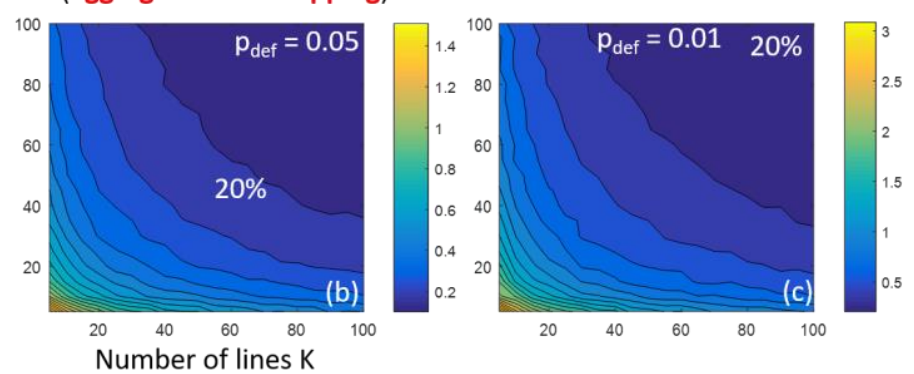

B2 (aggregated- nonoverlapping)

Number of lines
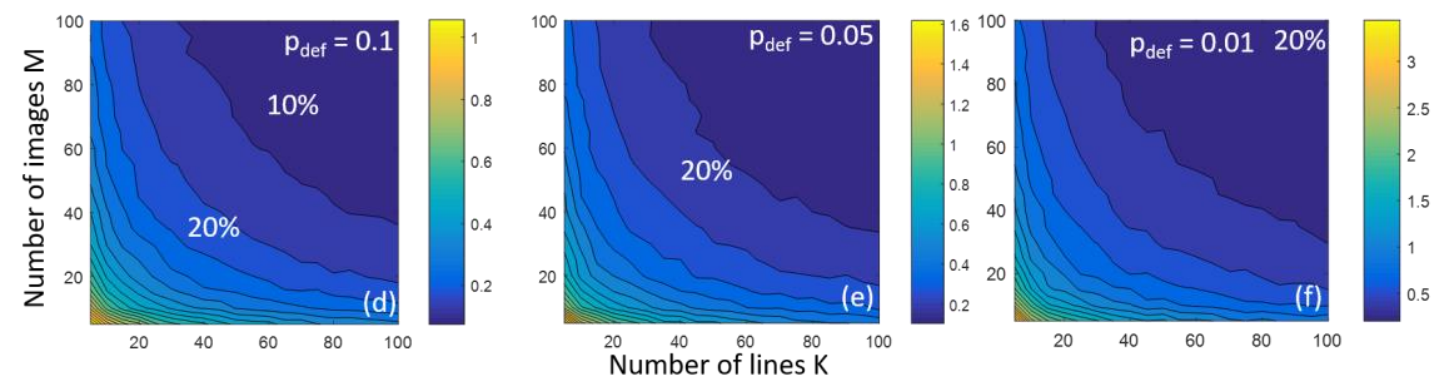

Fig. 5. Contour graphs of the normalized standard deviation of the fraction of missing lines versus the number M of acquired SEM images and the number $\mathrm{K}$ of lines contained in these when the defects have positively correlated positions i.e. they appear in aggregations randomly distribution in the pattern. The mean size of the defect aggregates is 5 . The first row (a-c) shows the results of our statistical modelling when the images may include the same lines (overlapping) (case B1) while the second (d-f) the results for the case B2 where the images are imposed to include different lines. The columns correspond to different fraction of defects in the whole pattern: $\mathrm{p}_{\mathrm{def}}=0.1$ $(\mathrm{a}, \mathrm{d}), \mathrm{p}_{\mathrm{def}}=0.05(\mathrm{~b}, \mathrm{e})$ and $\mathrm{p}_{\mathrm{def}}=0.01(\mathrm{c}, \mathrm{f})$.

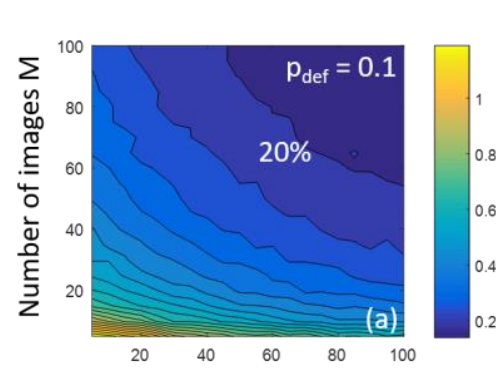
B1 (aggregated - overlapping)
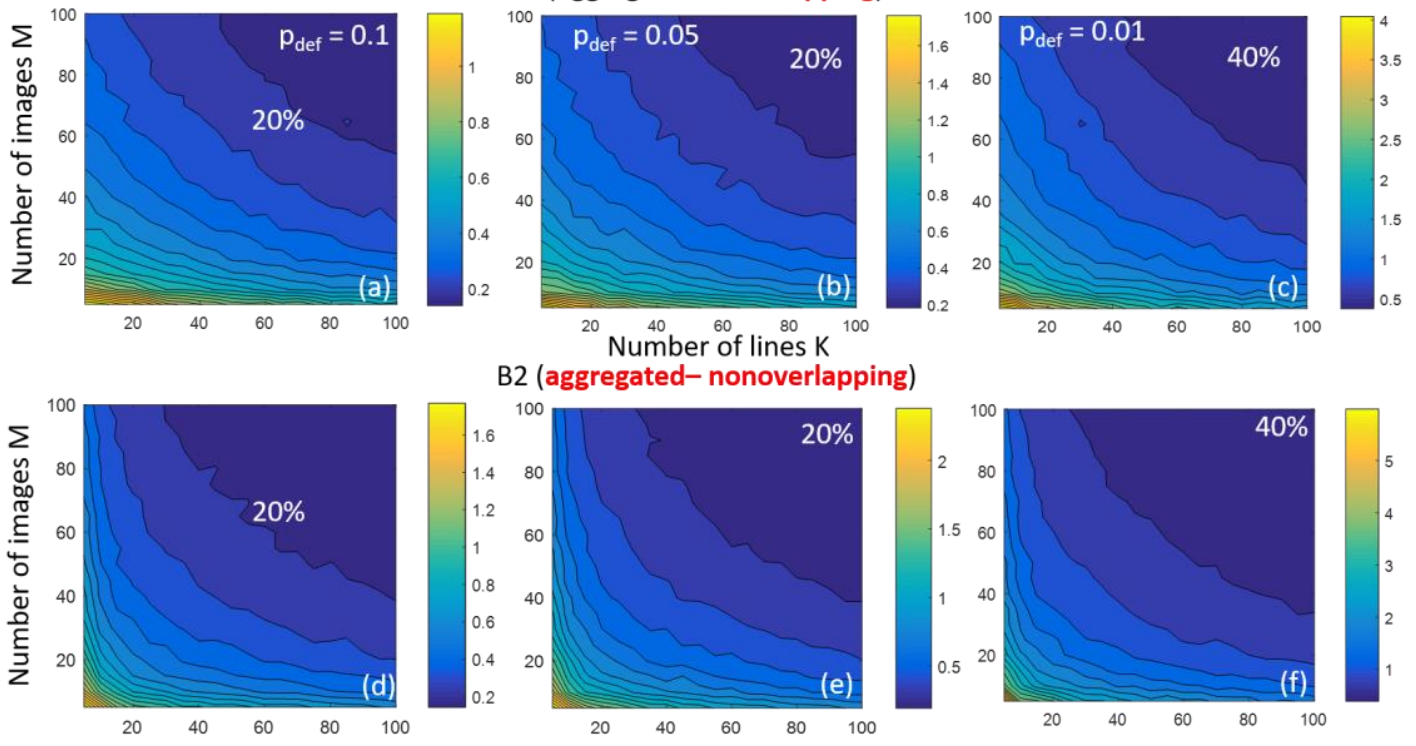

B2 (aggregated- nonoverlapping)

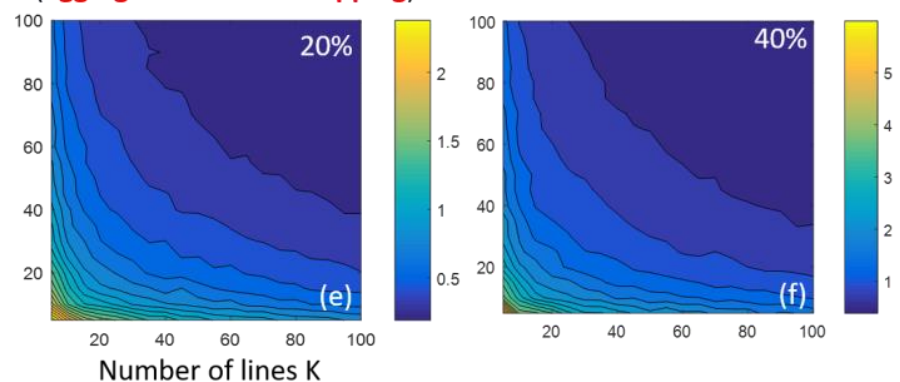

Fig. 6. Contour graphs of the normalized standard deviation of the fraction of missing lines versus the number M of acquired SEM images and the number $\mathrm{K}$ of lines contained in these when the defects have positively correlated positions i.e. they appear in aggregations randomly distribution in the pattern. The mean size of the defect aggregates is 20 . The first row (a-c) shows the results of our statistical modelling when the images may include the same lines (overlapping) (case B1) while the second (d-f) the results for the case B2 where the images are imposed to include different lines. The columns correspond to different fraction of defects in the whole pattern: $p_{\text {def }}=0.1$ $(\mathrm{a}, \mathrm{d}), \mathrm{p}_{\mathrm{def}}=0.05(\mathrm{~b}, \mathrm{e})$ and $\mathrm{p}_{\mathrm{def}}=0.01(\mathrm{c}, \mathrm{f})$. 


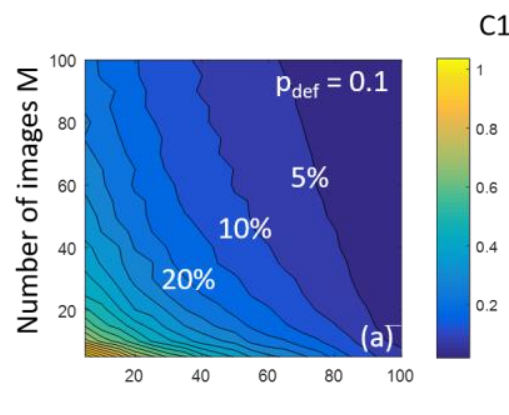

C1 (periodic-like - overlapping)
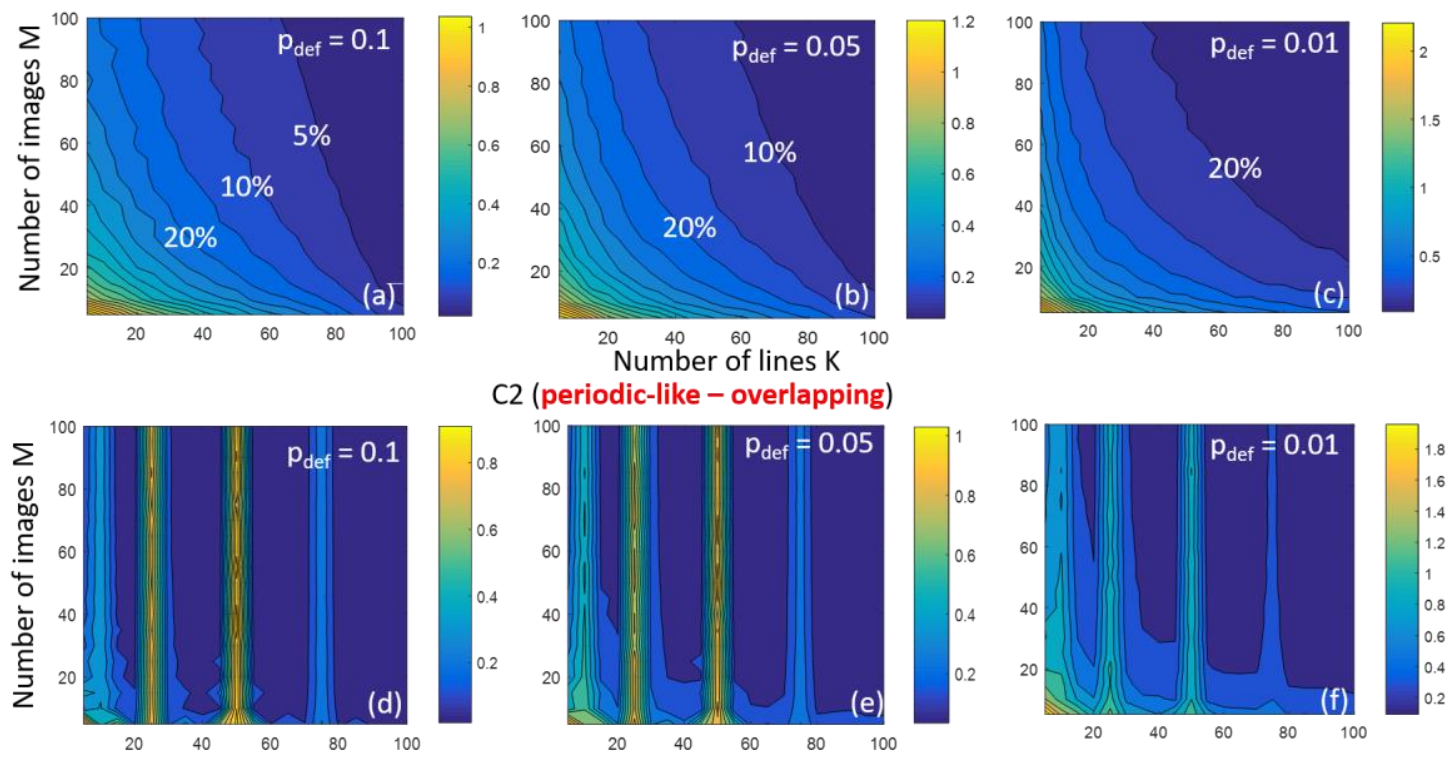

C2 (periodic-like - overlapping)
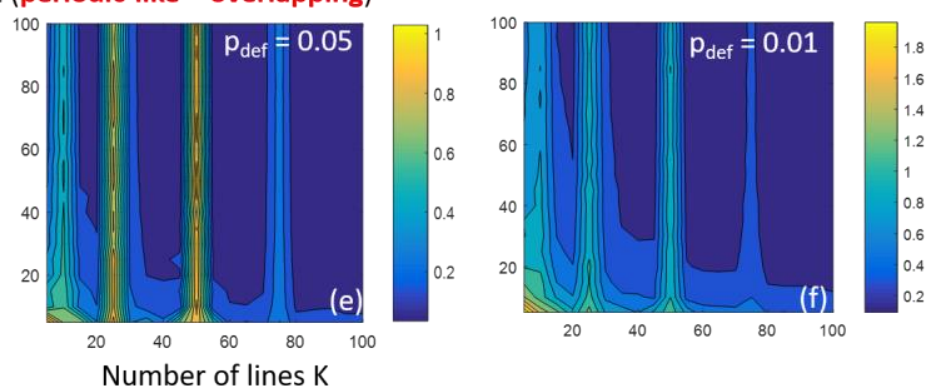

Fig. 7. Contour graphs of the normalized standard deviation of the fraction of missing lines versus the number M of acquired SEM images and the number $\mathrm{K}$ of lines contained in these when the defects have negatively correlated positions i.e. they appear in periodiclike arrangements in the pattern. The period is approximately 100 pitches. The first row (a-c) shows the results of our statistical modelling when the images may include the same lines (overlapping) (case B1) while the second (d-f) the results for the case B2 where the images are imposed to include different lines. The columns correspond to different fraction of defects in the whole pattern: $\mathrm{p}_{\mathrm{def}}=0.1(\mathrm{a}, \mathrm{d})$, $\mathrm{p}_{\mathrm{def}}$ $=0.05(\mathrm{~b}, \mathrm{e})$ and $\mathrm{p}_{\mathrm{def}}=0.01(\mathrm{c}, \mathrm{f})$.

\section{SUMMARY AND FUTURE WORK}

In this work, we examined the metrology of defects in line patterns when they have the form of missing lines. The main question we addressed has been the accuracy of the measurement of their fraction when SEM microscopy is used with images of limited range. The method we developed to attack the problem has been the computational modeling of the statistics of SEM measurements emphasizing the role of the parameters available in SEM user. Special attention has been paid on the interplay between metrological design and pattern defects characteristics such as the correlations of their positions in pattern. The motivation for the consideration of correlations in defects has been raised by the reduced size of pattern features in scales where the source of defects can affect more than one missing line.

The computational modeling we elaborate reveals significant effects of defect correlations on metrology output. Whereas for uncorrelated defects, the modeling predicts a behavior expected from sampling theory of large data sets (no significant effect of the way that images are acquired and a symmetry between number of image and lines since the most critical parameter seems to be the total number of lines included in the analysis of all images.

The correlations between defects introduce first a differentiation in the way of image acquisition is realized since the modeling predicts slight different results. Apart from this, the correlations break down the symmetry between number of images and lines concerning their effects on defect fraction uncertainty. Quite interestingly, the positive (negative) correlations favour the increase of images (lines) in the measurement protocol. 


\section{REFERENCES}

1. Higashiki, T., et. Al., "Nanoimprint lithography and future patterning for semiconductor devices", J. Micro/Nanolith. MEMS MOEMS 10(4), 043008 (2011)

2. Chen et al., "Defect control in NIL" Journal of Vacuum Science \& Technology B: Microelectronics and Nanometer Structures Processing, Measurement, and Phenomena 23, 2933 (2005);

3. Zhu et al., "Scatterometry for nanoimprint lithography" Journal of Vacuum Science \& Technology B 34, 06K503 (2016);

4. Martin Kreuzer et al., "In-line metrology for roll-to-roll UV assisted nanoimprint lithography using Diffractometry", APL Materials 6, 058502 (2018);

5. Schulz, K., et al. "SEM AutoAnalysis: enhancing photomask and NIL defect disposition and review" Proc. SPIE, Volume 10446, 33rd European Mask and Lithography Conference; 104460I (2017)

6. Keenan, D., "A Time Series Analysis of Binary Data”, Journal of the American Statistical Association Vol. 77, No. 380 (Dec., 1982), pp. 816-821 